\title{
Квалификация ВЭЖХ-оборудования Тестовые испытания колонок для гидрофильной и ионной хроматографии на основе силикагеля
}

Е. А. Окунская, К. С. Сычев, к.Х. н., Integrated BioSeparation Solutions, Эстония sales@hplc.today
УДК 543.544.5.068.7

Статья поступила в редакцию 15.08.2019

Статья принята к публикации 29.08.2019

\begin{abstract}
Предложен оригинальный метод тестирования полярных колонок на основе силикагеля в условиях реализации ионной, гидрофильной и смешанной ионной-гидрофильной ВЭЖХ. Он позволяет оценивать и сравнивать физико-химические и эксплуатационные характеристики колонок: удерживание по гидрофильному механизму, знак и величину заряда ионита, определяющих удерживание в ионной хроматографии и селективность в смешанной ионной-гидрофильной ВЭЖХ, химическую инертность полярных неподвижных фаз на основе силикагеля и эффективность упаковки полярных колонок. Обсуждаются условия, при которых можно успешно применять предложенный тест для квалификации хроматографических колонок для ионной и гидрофильной ВЭЖХ на основе силикагеля.
\end{abstract}

\section{ВВЕДЕНИЕ}

В первой статье серии авторы сформулировали термин "квалификация хроматографической колонки" для обозначения высокоинформативных тестов ВЭЖХ-колонок, позволяющих наиболее полно описать их физико-химические и эксплуатационные свойства: величину удерживания по доминирующему механизму, селективность, химическую инертность, эффективность". Также был предложен оригинальный тест для квалификации обращенных и смешанных фаз с доминированием обращенно-фазового механизма, с помощью которого можно установить все перечисленные характеристики. При этом инновационной составляющей было разделение селективности на два ортогональных параметра, связываемых с примесями минорных ВЭЖХ-режимов: гидрофильного (HILIC) и переносно-зарядного (CT).

Данная работа посвящена описанию второго оригинального теста ВЭЖХ-колонок, который предназначен для квалификации любых полярных колонок на основе силикагеля, применяемых в комбинации

Окунская Е.А., Сычев К. С., Стыскин И. Е. Квалификация ВЭЖХоборудования. Тестовые испытания обращенно-фазовых колонок. АНАЛИТИКА. 2017. № 4. С. 82-87. с водно-органическими подвижными фазами. В этом случае механизм удерживания всегда представляет собой смесь двух хроматографических режимов: гидрофильного и ионного.

И ионная, и гидрофильная хроматографии - два сильно неоцененных вида ВЭЖХ для фармацевтических приложений. Упрощенно можно считать, что удерживание в ионном режиме определяется зарядом адсорбата, а в гидрофильном - общим числом заряженных групп в его составе. Учитывая, что большинство фармацевтических препаратов - это заряженные соединения (органические соли), то область применения для гидрофильной и ионной хроматографии в фармацевтике, биотехнологии, химии природных соединений должна быть, по крайней мере, не уже, чем для обращенно-фазовой хроматографии.

Тем не менее на практике новые гидрофильные и ионные методы разрабатываются пока сравнительно нечасто, а внедряются и успешно применяются - еще реже. На это есть целый ряд причин, совершенно не связанных с самими методами, но имеющих прямое отношение к недостаточному пониманию аналитиками их особенностей и "подводных камней".

К этому же ряду причин можно причислить и отсутствие у аналитика инструментов, которые позволили бы 
сравнивать физико-химические свойства гидрофильных и ионных неподвижных фаз различных типов и производителей, а также судить об их качестве и воспроизводимости свойств от партии к партии.

Цель работы состоит в том, чтобы продемонстрировать возможность квалификации применяемых на практике гидрофильных и ионных ВЭЖХ-колонок на основе силикагеля с помощью теста, разработанного специалистами нашей компании.

\section{ЭКСПЕРИМЕНТАЛЬНАЯ ЧАСТЬ}

Соотношение двух составляющих смешанного гидрофильноионного механизма в каждом конкретном случае определяется как химией неподвижной фазы и составом подвижной фазы, так и свойствами адсорбатов, из которых составлена тестовая смесь. Тем не менее основной параметр, контролирующий это соотношение - доля ацетонитрила в подвижной фазе. Вклад ионного механизма растет с ее уменьшением, а вклад гидрофильного механизма растет с ее увеличением.

Таким образом, содержание ацетонитрила в подвижной фазе контролирует не только (и не столько) удерживание, сколько, в первую очередь, селективность разделения. Варьирование доли ацетонитрила вызывает наиболее выраженное изменение селективности с изменением порядка элюирования в том случае, когда адсорбаты содержат и основные, и кислотные функциональные группы. Действие этого фактора более мягкое, если тестовая смесь состоит из одноосновных органических оснований и кислот, и почти не выражено, если тестовая смесь состоит из органических ионов с одинаковым знаком заряда.

Из этого наблюдения следуют два вывода, важных для разработки тестовой системы. Во-первых, выборка адсорбатов не должна содержать амфотерных органических соединений, поскольку это привело бы к недостаточной робастности тестового разделения и, соответственно, неустойчивости самого теста. С другой стороны, выборка не может состоять только из соединений одного знака заряда, поскольку такой тест мог бы работать только для одного типа ионита (катионита или анионита). Кроме того, среди тестовых адсорбатов должны быть и нейтральные соединения, по которым можно было бы судить об удерживании только по гидрофильному механизму.

Таким образом, тестовая смесь должна состоять из гидрофильных нейтральных соединений, органических оснований и органических кислот.

Во-вторых, тест должен проводиться в условиях, которые априори гарантировали бы смешанный режим с сопоставимыми по величине вкладами обоих механизмов - гидрофильного и ионного. Сопоставимость теста, как со слабыми, так и с сильными ионитами, требует значения рН буфера, близкого к нейтральному.
Что касается доли ацетонитрила в подвижной фазе, то она не должна быть слишком низкой, порядка 50\% или менее, поскольку в подобных условиях гидрофильный механизм подавляется почти полностью. Существенное влияние гидрофильного механизма для удерживания однозарядных органических ионов начинается приблизительно от 70\%. В то же время содержание ацетонитрила не должно быть слишком высоким, 85\% и более, поскольку в таких условиях подавляется ионный механизм, а форма пиков ионных соединений недопустимо искажается.

В результате, для проведения тестирования была выбрана подвижная фаза, состоящая из ацетонитрила и 20-мМ водного раствора формиата аммония, смешанных в объемном отношении $80: 20$.

В тестовую смесь включили следующие соединения (в порядке элюирования на катионите): (1) сахарин, (2) бензойная кислота, (3) кофеин, (4) аскорбиновая кислота, (5) пиридоксин, (6) серотонин. Кофеин - нейтральное соединение; сахарин, бензойная кислота и аскорбиновая кислота - кислоты, пиридоксин и серотонин - основания.

Детектирование проводили при помощи диодноматричного или двухволнового сканирующего УФ-детектора на двух длинах волн - 230 нм (синяя линия на рисунках) и 270 нм (красная линия на рисунках).

Для каждой тестируемой колонки необходимо предварительно измерить нулевое (мертвое) время; в нашей работе для этих целей применяли нафталин.

\section{ОБСУЖДЕНИЕ РЕЗУЛЬТАТОВ}

На рис. 1 приведены три хроматограммы тестовой смеси, полученные на сильном катионите, слабом катионите и слабом амионите. На катионитах наиболее удерживаемым адсорбатом является серотонин - сильное основание. На анионитах последний пик - всегда аскорбиновая кислота, одинаково сильно удерживающаяся как по анионообменному, так и по гидрофильному механизмам.

На сильном катионите сахарин элюируется до нулевого времени, что не является ошибкой - в этом случае вклад ионной эксклюзии для сахарината превосходит его удерживание по гидрофильному механизму.

В целом, закономерность в порядке элюирования адсорбатов хорошо прослеживается: при переходе от сильного катионита к слабому, а затем к аниониту удерживание оснований возрастает, а удерживание кислот падает - из-за влияния ионного механизма.

При этом на удерживание кофеина влияет только гидрофильность применяемой неподвижной фазы, но не ее заряд.

Таким образом, коэффициент удерживания кофеина может служить параметром для оценки удерживающей способности неподвижной фазы по гидрофильному механизму безотносительно ее заряда. 
В то же время для оценки знака и величины заряда полярной неподвижной фазы был применен параметр, вычисляемый следующим образом:

$$
\begin{gathered}
\text { Фактор заряда }=\left(t_{\mathrm{R}}(\text { серотонин })-\right. \\
\left.t_{\mathrm{R}}(\text { сахарин })\right) / t_{0}
\end{gathered}
$$

где $t_{\mathrm{R}}$ (серотонин) - время удержива-

ния серотонина,

$t_{R}($ сахарин $)$ - время удерживания сахарина,

$t_{0}$ - нулевое время.

На рис. 2 приведен график, каждая точка на котором соответствует определенной неподвижной фазе; по оси абсцисс отложен фактор заряда, вычисленный по приведенной выше формуле, а по оси ординат - коэффициент удерживания кофеина.

Из графика хорошо видно, что нулевой заряд подвижной фазы соответствует значению фактора заряда около единицы; анионитам (положительный заряд) соответствует область значений менее единицы, катионитам (отрицательный заряд) - более единицы.

Таким образом, тест можно применять для сравнения гидрофильности различных полярных неподвижных фаз, а также величин заряда катионитов и анионитов.

Еще одно преимущество теста возможность оценки и сравнения качества полярных колонок различных производителей. Химическую инертность целесообразно оценивать по коэффициенту асимметрии пиридоксина. На "загрязненных" примесями металлов, некачественных силикагелях пик пиридоксина становится асимметричным, в то время как на чистых, качественных силикагелях он симметричен. Эффективность упаковки колонки можно проверять по пику аскорбиновой кислоты (см. рис. 1).

К сожалению, предложенный тест не применим для характеризации смешанных обращенно-ионных неподвижных фаз с неполярным эндкеппингом. На рис. 3 приведен пример хроматограммы тестовой смеси,
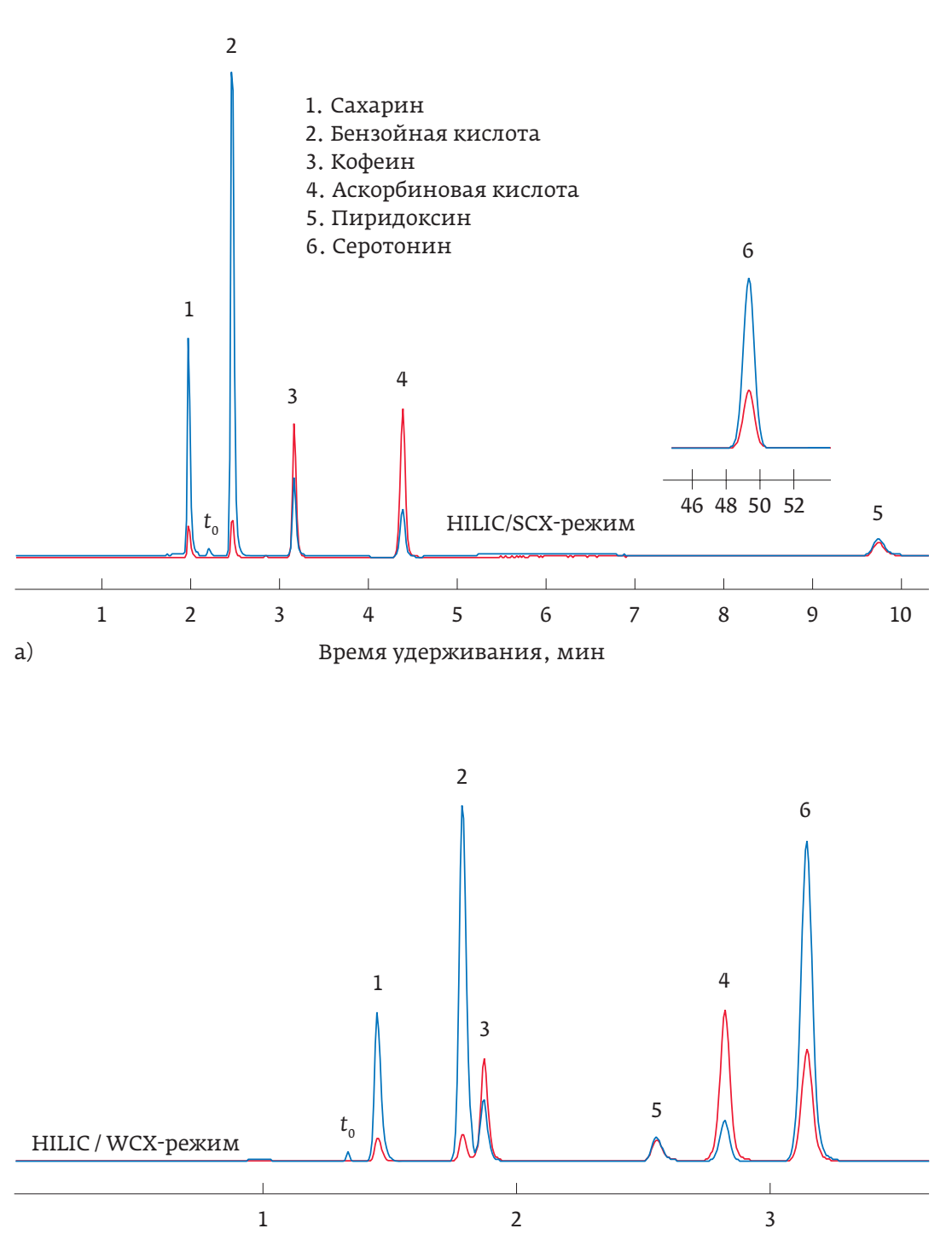

б)

Время удерживания, мин

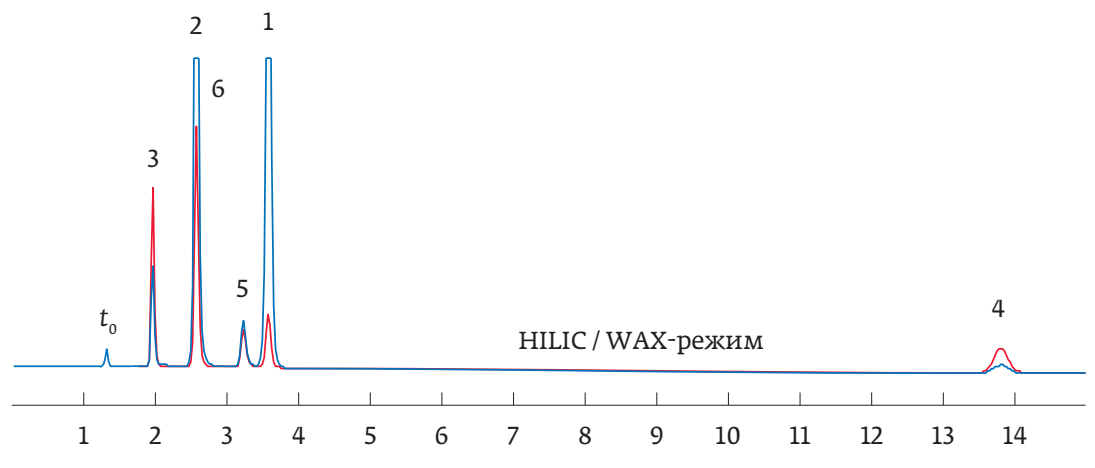

в)

Время удерживания, мин

Puc. 1. Хроматограмма тестовой смеси, полученной на сильном катионите (a), слабом катионите (б) и слабом амионите (в) 


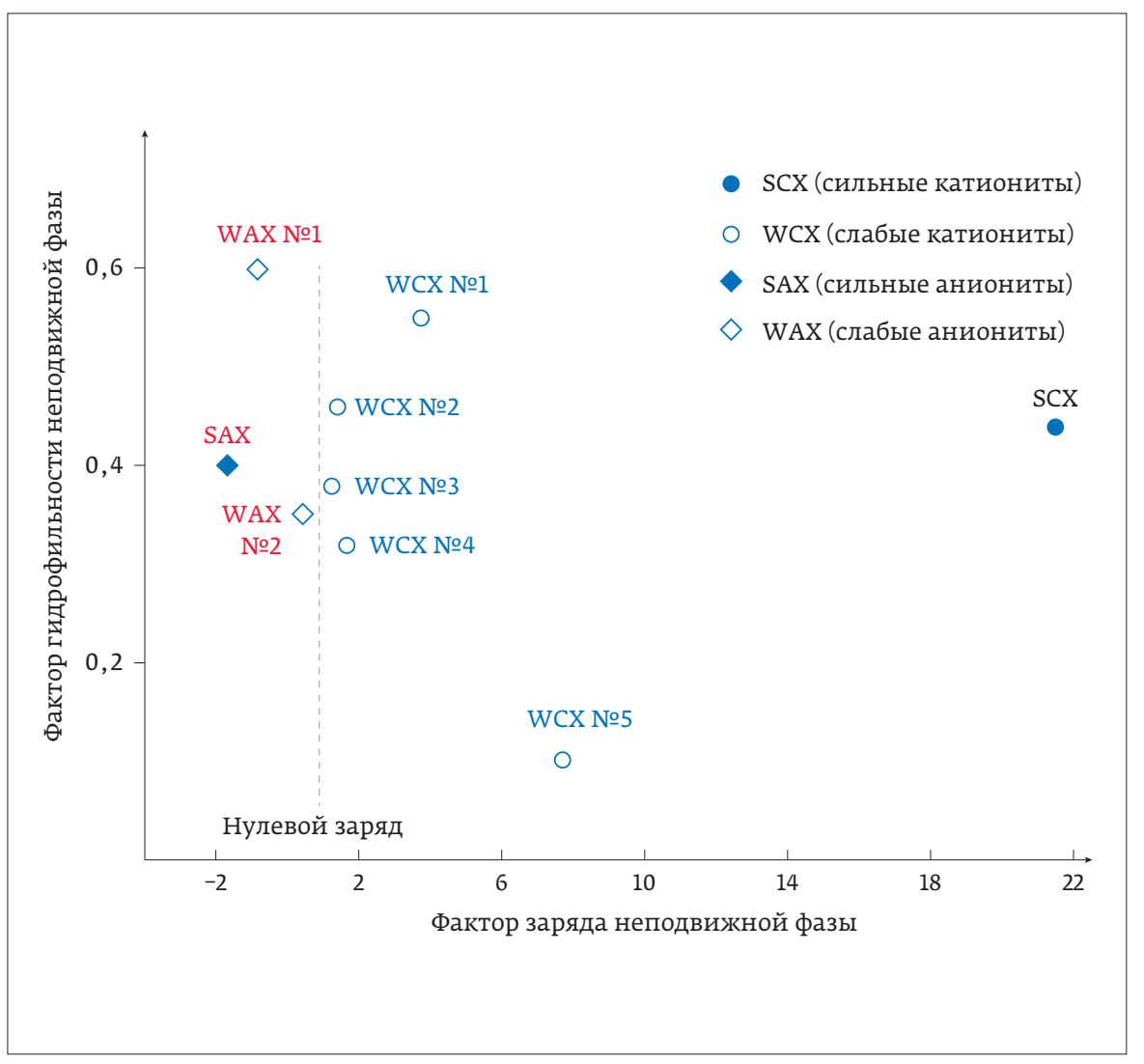

Puc. 2. Классификация полярных фаз на основе силикагеля различных типов и производителей согласно их гидрофильности и заряда

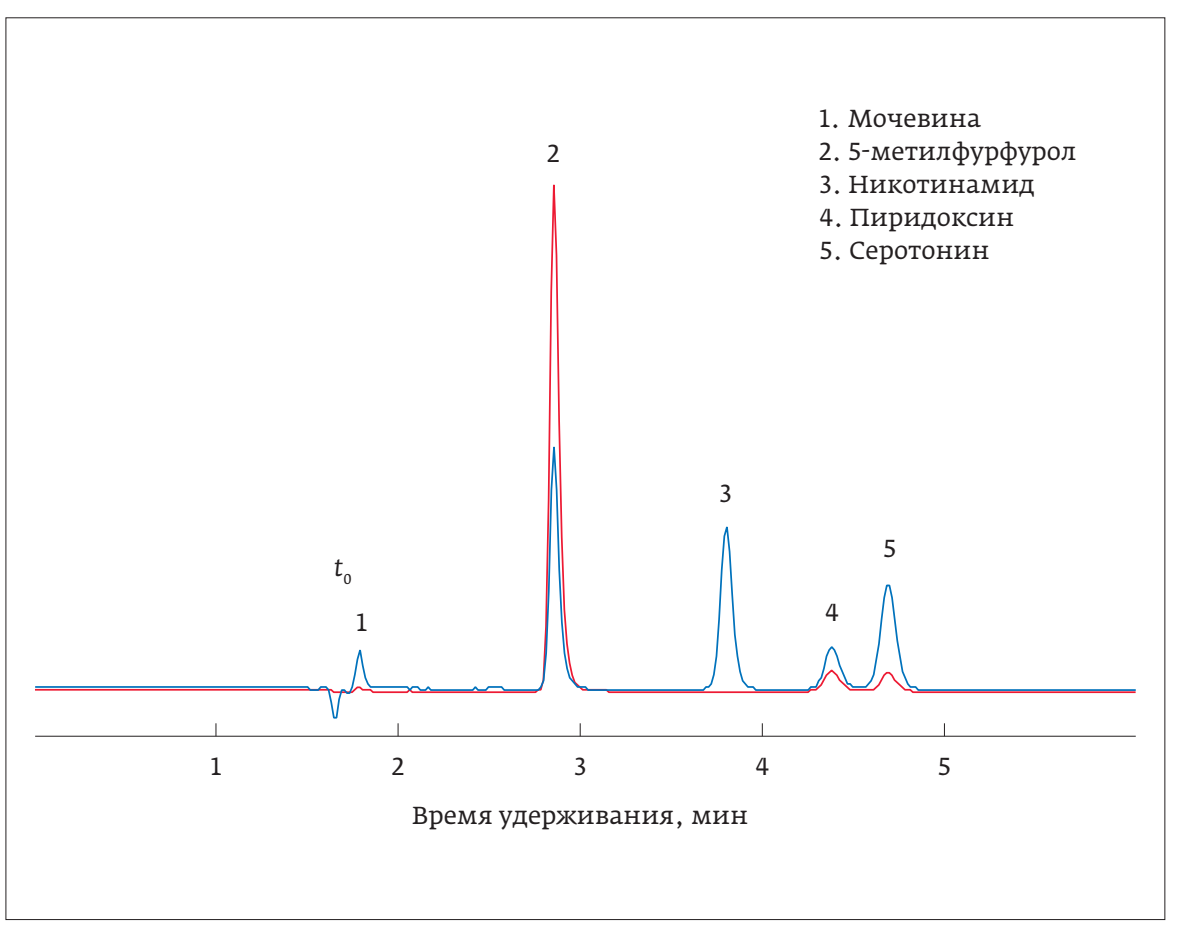

Puс. 3. Хроматограмма тестовой смеси на смещанной обращенной-катионообменной неподвижной фазе которая может применяться для квалификации смешанных обращенныхкатионообменных фаз такого типа.

Смесь включает мочевину, 5-метилфурфурол, никотинамид и пиридоксин (слабые основания), серотонин (сильное основание). Подвижная фаза состоит из ацетонитрила и 50-мМ водного раствора однозамещенного фосфата аммония, смешанных в объемном отношении $23: 77$.

Величину удерживания по обращенно-фазовому механизму можно оценить по коэффициенту удерживания 5-метилфурфурола, эффективность упаковки - по пику 5-метилфурфурола, величину положительного заряда - по коэффициенту удерживания никотинамида, химическую инертность - по коэффициенту асимметрии пика пиридоксина. Нулевое время можно измерять по любому нейтральному гидрофильному соединению, например, по мочевине.

\section{выводы}

Применение оригинального метода тестирования полярных колонок на основе силикагеля в условиях реализации ионной, гидрофильной и смешанной ионной-гидрофильной ВЭЖХ позволяет оценивать и сравнивать их физико-химические и эксплуатационные характеристики:

- удерживание по гидрофильному механизму,

- знак и величину заряда ионита, определяющих удерживание в ионной хроматографии и селективность в смешанной ионнойгидрофильной ВЭЖХ,

- химическую инертность полярных неподвижных фаз на основе силикагеля,

- эффективность упаковки полярных колонок.

Тест пригоден для квалификации хроматографических колонок для ионной и гидрофильной ВЭЖХ на основе силикагеля. 